\title{
L'amplification des séquences de nucléotides par PCR et les nouvelles techniques de diagnostic moléculaire
}

\author{
M Goossens \\ Laboratoire de Génétique Moléculaire, Service de Biochimie, INSERM U91, \\ Hôpital Henri-Mondor, 94010 Créteil, France
}

(28e Réunion de la Société Française pour l'Étude de la Fertilité; Paris, 19-21 octobre 1989)

\begin{abstract}
Résumé - La PCR (polymerase chain reaction) est une méthode qui permet d'amplifier sélectivement un segment d'ADN ou d'ARN mesurant jusqu'à 2000 paires de bases (pb). Des cycles répétés d'allongement enzymatique d'amorces oligonucléotidiques sont effectués en présence de précurseurs de la synthèse d'ADN el d'une enzyme thermostable, une ADN polymérase extraite de la bactérie Thermus aquaticus (Taq polymérase). Les amorces encadrent la séquence d'intérêt, et l'allongement se fait dans des directions opposées, de sorte que plusieurs centaines de milliers de copies de cette séquence sont générées en quelques heures, permettant par là d'effectuer un véritable clonage moléculaire. La réaction est simple, sensible, et l'ADN, ou l'ARN, contenu dans une seule cellule est théoriquement suffisant pour qu'une séquence spécifique soit détectée. Cette méthode est maintenant largement utilisée en biologie moléculaire fondamentale et appliquée, et prend une place de plus en plus grande dans les approches diagnostiques.
\end{abstract}

PCR ampliflcation / ADN / ARN / diagnostic moléculalre

Summary - PCR amplification of nucleotidlc sequences and new dlagnostic techniques. The polymerase chain reaction (PCR) is a method for selective amplification of DNA or RNA segments of up to 2 kilobase pairs (kb) or more in length. Repeated cycles of enzymatic primer extension in opposite directions are performed using synthetic oligonucleotides flanking the sequences of interest and the heat-stable DNA polymerase from the archebacterium Thermus aquaticus (Taq polymerase). The reaction is simple, fast and extremely sensitive, and the DNA or RNA content of a single cell is sufficient to detect a specific sequence. This method has proved useful in simplifying complex analytical protocols in basic and applied molecular biology.

PCR amplification / DNA / RNA / molecular dlagnosis

\section{INTRODUCTION}

Le succès des méthodes courantes d'analyse des séquences d'ADN dépend de la possibilité de disposer de ces séquences en relativement grande quantité, de l'ordre de $10^{5}$ a $10^{6}$ molécules d'ADN ou d'ARN par test. Il est possible d'obtenir un grand nombre de copies d'une séquence donnée par les approches maintenant bien standardisées de clonage moléculaire dans des vecteurs bactériens ou viraux. Néanmoins, ces manipulations sont lourdes, fastidieuses et requièrent plusieurs semaines de travail, et elles ne sont donc pas adaptées aux problèmes que pose la mise en œuvre des tests de diagnostic exigeant une réponse rapide. Ceci explique que de 
nombreux efforts ont été engagés dans des recherches destinées à amplifier le pouvoir résolutif des méthodes actuelles et, parallèlement, à concevoir de nouvelles techniques propres à détecter de petits nombres de molécules. Ces efforts ont récemment abouti, et la plupart des techniques utilisées dans l'analyse des génotypes en biologie et en médecine comportent maintenant une étape très rapide d'amplification enzymatique des séquences d'ADN qui constitue un véritable "clonage moléculaire acellulaire". Ce processus révolutionnaire, appelé polymerase chain reaction (PCR) a été inventé il y a 4 ans par les chercheurs de la firme de biotechnologie californienne Cetus (Saïki et al, 1985; Mullis et Faloona, 1987). Parce qu'elle permet d'obtenir rapidement, avec facilité et en grande quantité les séquences d'ADN que l'on désire étudier, cette approche permet de renouveler les stratégies d'analyse des génomes, et est appliquée maintenant dans une gamme de plus en plus étendue d'études de biologie moléculaire.

\section{LES APPORTS DE L'AMPLIFICATION PAR PCR}

\section{Une nouvelle façon de cloner I'ADN}

L'approche classique de clonage de l'ADN, si elle s'est affranchie d'un certain nombre de difficultés initiales, est encore une entreprise techniquement lourde et fastidieuse, et reste du ressort du laboratoire de recherche. Dans cette approche, I'ADN chromosomique extrait des cellules nucléées est coupé par une endonucléase spécifique (enzyme de restriction) le fragmentant en plusieurs millions de morceaux de taille variable qui sont ensuite insérés individuellement dans une autre molécule
d'ADN - le vecteur - un plasmide bactérien le plus souvent. Ces molécules chimériques d'ADN recombinant sont introduites dans des bactéries dont les colonies, après isolement sur boîte de Pétri, constitueront autant de clones qu'il faudra ensuite caractériser, à la recherche du bon candidat.

Dans un grand nombre de situations, cette opération est maintenant avantageusement remplacée par lamplification par PCR. Cette technique procure une nouvelle façon de cloner enzymatiquement, in vitro, une séquence d'ADN bien définie. Lorsque cette approche est possible, le clone est obtenu en quelques heures au lieu des jours ou des semaines requis par les méthodes conventionnelles, d'où son intérêt dans la mise en œuvre des tests diagnostiques. La détection d'une séquence très faiblement représentée à l'aide d'une sonde, radioactive ou non, peut être grandement facilitée si on l'amplifie au préalable. Le produit de ce clonage enzymatique peut être analysé comme le serait un segment d'ADN cloné, tirant parti de tous les avantages offerts par la disponibilité, en grandes quantités, de I'ADN cible.

\section{Pourquoi amplifier ?}

Le génome haploïde humain contient quelques $3 \times 10^{9}$ paires de bases $(\mathrm{pb})$, et un fragment d'ADN mesurant 300 pb"en constitue donc une infime partie $\left(1 / 10^{7}\right)$. Dans l'état actuel de la technique, une séquence de quelques centaines de nucléotides peut être amplifiée au moins un million de fois, ce qui fait que partant d'un microgramme d'ADN génomique total, il est possible en quelques heures d'obtenir la même quantité, un microgramme, du fragment cible amplifié. Afin de fixer les idées, quelles sont les quantités d'ADN présentes dans les 
cellules nucléées de divers échantillons biologiques susceptibles d'être analysés ? Une cellule contient environ $7 \mathrm{pg}\left(7 \times 10^{-6}\right.$ $\mu \mathrm{g})$ d'ADN, une goutte de sang $1,5 \mu \mathrm{g}$, une goutte de sperme $10 \mu \mathrm{g}$, et d'un cheveu peut être obtenu $0,3 \mu \mathrm{g}$ ou $10^{-4} \mu \mathrm{g}$ selon qu'il a été arraché ou qu'il est tombé de luimême. Par rapport à la méthode courante d'analyse des gènes (le Southern blot) qui requiert un minimum de $5 \mu \mathrm{g}$ d'ADN, l'amplification des séquences d'ADN (ou d'ARN) permet de travailler sur une tache de sang, de sperme, sur quelques cellules buccales desquamées, autorisant la mise en œuvre de tests extrêmement sensibles et dont les applications sont multiples : dépistage génétique (Amselem et al, 1988; Gyllensten et Erlich, 1988) et typages individuels, détection des séquences à l'échelle unicellulaire (Jeffreys et al, 1988; Li et al, 1988), détection des cellules malignes dans les bilans d'extension en cancérologie (Lee et al, 1987; Crescenzi et al, 1988; Kawasaki et al, 1988; Pignon et al, 1989), médecine légale, police scientifique (Bugawan et al, 1988; Higuchi et al, 1988), etc. Bien entendu, la très grande sensibilité de ce processus expose à des risques d'erreur, notamment par contamination de l'échantillon avant ou pendant l'analyse, et l'enjeu de ces applications est tel qu'il est absolument nécessaire de tout mettre en œuvre pour se prémunir contre ce risque (voir plus loin).

\section{LA TECHNIQUE}

\section{La cible, l'enzyme et les amorces}

Des millions de copies du même segment d'ADN sont synthétisées pendant plusieurs cycles (25 à 30 le plus souvent) au cours desquels se succèdent dénaturation de I'ADN, hybridation des amorces spécifi- ques aux matrices d'ADN simple brin alors disponibles, et allongement de ces amorces par polymérisation enzymatique de précurseurs désoxyribonucléotidiques (fig 1). Au cours de chacun des cycles, la quantité du segment d'ADN cible double, le produit d'amplification s'accumulant de façon exponentielle. L'enzyme utilisée est une ADN polymérase active à haute température (Taq polymérase), extraite de la bactérie thermophile Thermus aquaticus. Les amorces, qui sont constituées de courts oligomères (20-25 nucléotides) complémentaires des séquences qui limitent de part et d'autre la cible, confèrent à la réaction sa spécificité : leur position et leur nature conditionnent la longueur du segment amplifié et son identité. II est difficile d'amplifier un fragment long de plus de 2 à 3000 nucléotides, et bien entendu la séquence que l'on veut ainsi produire en grande quantité doit être au préalable connue. Chaque étape d'un cycle s'effectue à une température appropriée, élevée pour dénaturer l'ADN, plus basse pour l'hybridation des amorces et la synthèse. La Taq polymérase, qui n'est pas inactivée pendant l'étape de dénaturation de l'ADN, fonctionne aux températures élevées nécessaires à la spécificité de la réaction d'hybridation aux amorces avec la cible. A la fin du processus est généré, malgré la petite taille de ces amorces, un produit d'amplification unique, constitué de plusieurs millions de copies de la séquence cible. Les cycles de réactions peuvent être effectués à l'aide d'un automate qui permet de programmer les différentes températures, la durée de chaque étape, le nombre de cycles, etc.

L'ARN peut également être amplifié. II suffit, au cours d'une étape préliminaire, de transformer la molécule d'ARN messager d'intérêt en un ADNc (ADN complémentaire) à l'aide d'une transcriptase inverse et d'une amorce adéquate (complémentaire 

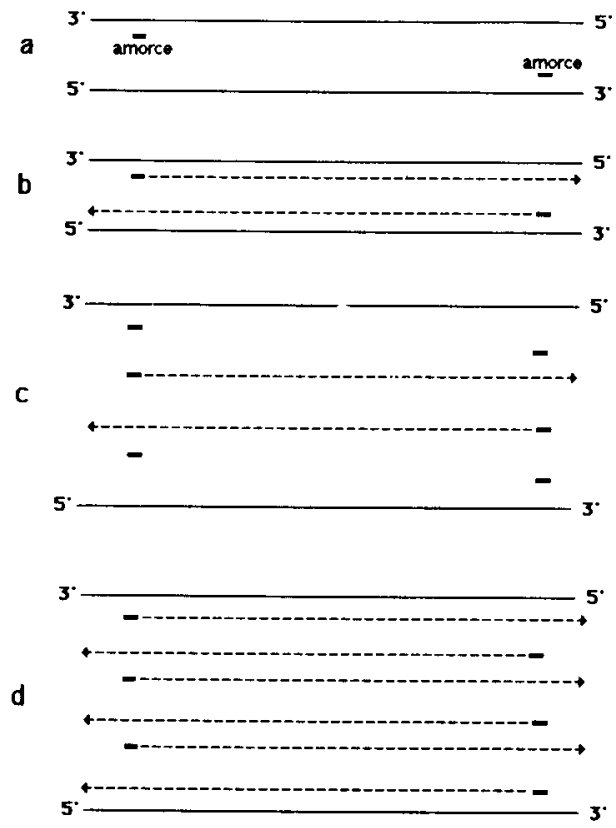

Fig 1. Principe de la réaction d'amplification par I'ADN polymérase. a) dénaturation et hybridation des amorces et b) élongation (1er cycle); $c$ ) dénaturation et hybridation des amorces et d) élongation $\left(2^{\theta} \mathrm{cycle}\right)$.

d'une séquence localisée du côté $3^{\prime}$ ). Ce ADNc sert ensuite de matrice à la synthèse d'un second brin qui est allongé à partir d'une amorce 5' et le duplex est amplifié comme décrit précédemment (Frohman et al, 1988).

II faut noter que durant le processus d'amplification, les amorces, qui sont en grand excès dans le milieu réactionnel et dont 2 sont consommées par molécule amplifiée à chaque cycle, sont physiquement incorporées dans le produit d'amplification. Ce produit est en effet constitué d'une séquence copiée sur chacune des matrices par l'ADN polymérase et des amorces qui encadrent, de part et d'autre, cette séquence. Ceci apporte donc des moyens nouveaux de modification de I'ADN : les amorces peuvent contenir des sites de restriction, facilitant les manipulations ultérieures du segment amplifié, ou des nucléotides artificiellement modifiés, sur lesquels ont été branchées des molécules comme par exemple la biotine, permettant la détection du produit à l'aide de réactions immuno-enzymatiques. Ailleurs, l'introduction dans l'une des amorces d'un promoteur viral pourra permettre, ultérieurement, de transcrire le produit amplifié en ARN dans des systèmes acellulaires appropriés. Enfin, on peut introduire des changements de nucléotides dans les amorces : comme celles-ci sont recopiées à chaque cycle, la molécule amplifiée sera différente de la matrice originale. Cette astuce est très utile pour faire de la mutagénèse in vitro (Rochiltz et al, 1988).

\section{Le renouvellement des techniques de diagnostic moléculaire}

L'amplification des séquences d'ADN par PCR est actuellement la méthode de choix pour détecter la présence (ou démontrer l'absence) d'une séquence donnée, et pour en analyser les variations ponctuelles soit délétères, soit neutres (polymorphismes). Ainsi peuvent être recherchés et analysés au nucléotide près un certain nombre de remaniements génomiques, qu'il s'agisse de délétions ou d'additions de matériel génétique (Saïki et al, 1986, 1988). De même, toute séquence appartenant au génome d'un micro-organisme pathogène et présente au sein d'un échantillon biologique sera aisément détectable (Byrne et al, 1988; Ou, 1988). Le succès de ces analyses est conditionné par le choix des amorces d'amplification et par les techniques d'analyse de l'ADN amplifié.

Parmi ces techniques, citons : 1) l'électrophorèse simple du produit d'amplifica- 
tion : elle peut révéler une modification de la taille attendue de ce produit, témoignant d'un remaniement, délétion ou addition. Sa coupure par une endonucléase de restriction avant l'électrophorèse permet de caractériser des polymorphismes nucléotidiques (restriction fragment length polymorphisms ou RFLP) ou des mutations ponctuelles altérant un des sites de restriction. La fluorescence en lumière UV de I'ADN traité au bromure d'éthidium permet d'observer directement les bandes, rendant souvent inutile l'hybridation avec des sondes spécifiques marquées, compte tenu de la grande quantité de produit d'amplification obtenue (Kogan et al, 1987); 2) la détection de mutations ponctuelles par hybridation de I'ADN fixé sur membranes (dot-blot) avec des sondes oligonucléotidiques spécifiques d'allèle, radiomarquées: ces oligosondes spécifiques sont les copies conformes de I'ADN normal et muté, et lorsqu'elles sont hybridées avec l'ADN à étudier, elles forment soit des homoduplex (ADN normal - sonde normale, ADN muté - sonde mutée), soit des hétéroduplex (ADN normal - sonde mutée ou l'inverse). Dans les conditions expérimentales choisies, seuls les produits d'hybridation homoduplex sont stables, ce qui permet de typer les mutations sans ambiguïté. Comme un grand nombre d'échantillons d'ADN peuvent être simultanément amplifiés et analysés de la sorte, cette approche est extrêmement efficace pour le dépistage à grande échelle des mutations dans les groupes de populations à risque pour une maladie génétique ou cancéreuse (Amselem et al, 1988; Diaz-Chico et al, 1988); 3) l'électrophorèse du produit en gels dénaturants : cette technique permet de mettre en évidence les variations nucléotidiques; celles-ci modifient le comportement fusionnel d'un segment d'ADN qui migre dans un gel d'électrophorèse contenant une concentration croissante d'agents dénaturants (urée, formamide). Dès que la molécule d'ADN s'est dissociée partiellement, elle s'ancre dans la matrice du gel et s'immobilise. Deux molécules ne différant que par un seul nucléotide vont donc arrêter leur migration à des positions différentes lorsque la substitution nucléotidique modifie la température de fusion du duplex. Toute différence de migration, objectivée directement par coloration spécifique des fragments d'ADN in situ, indique l'existence d'une mutation dont la caractérisation peut être ensuite effectuée par séquençage direct du produit d'amplification (Myers et al, 1987; Attree et al, 1989); 4) le séquençage du produit d'amplification : il est relativement aisé de déterminer la séquence des fragments "clonés" enzymatiquement, en adaptant légèrement les techniques éprouvées de séquençage (Wong et al, 1987; Wrischnick et al, 1987; Engelke et al, 1988).

\section{LES APPLICATIONS}

Elles sont très nombreuses, dans le domaine de la recherche fondamentale ou au niveau des applications médicales. Seules seront évoquées ici les possibilités nouvelles qu'elles apportent en génétique.

\section{Le diagnostic des maladies génétiques}

Les techniques évoquées plus haut permettent, une fois qu'un locus spécifique est amplifié, de distinguer les différents allèles normaux et délétères et éventuellement de caractériser les mutations. La rapidité de mise en œuvre de la PCR et sa grande sensibilité se prêtent bien à l'analyse individuelle et au dépistage de masse. Les tests de diagnostic anténatal d'un nombre croissant de maladies monogéniques reposent maintenant sur la technologie PCR, qu'il s'agisse de maladies à gène caractérisé 
(drépanocytose, thalassémies, hémophilies, myopathie de Duchenne, mucoviscidose, etc), ou d'affections dont seule la position chromosomique du locus est connue. Les mutations ponctuelles et certaines délétions peuvent être directement caractérisées lorsque le gène étudié est de petite taille. L'étendue du gène de la dystrophine $(2000 \mathrm{pb})$ rend difficile l'analyse directe des délétions responsables de la myopathie de Duchenne. Néanmoins, ces délétions peuvent être criblées grâce à l'utilisation d'un processus d'amplification dit «multiplex": les régions qui sont le siège potentiel de ces délétions sont simultanément amplifiées au cours de la même réaction, à l'aide d'un mélange d'amorces judicieusement choisies pour générer des produits d'amplification de longueurs différentes. Après l'électrophorèse des multiples produits d'amplification obtenus, la disparition ou le déplacement d'une ou plusieurs bandes objective l'existence d'une délétion de matériel génétique (Chamberlain et al, 1988). Lorsque les mutations ne peuvent être directement identifiées, la présence du gène anormal peut être déduite de l'analyse de marqueurs RFLPs liés. II suffit, dans ce cas, d'amplifier le segment d'ADN qui contient le site de restriction polymorphe et d'analyser par électrophorèse le produit d'amplification après coupure enzymatique (Feldman et al, 1988).

Récemment, la possibilité d'analyser les séquences d'ADN à partir d'une seule cellule a ouvert la voie à l'étude, avant l'implantation, des embryons fécondés in vitro. Le sexe d'un embryon a ainsi été étudié à l'aide d'une sonde nucléique spécifique du chromosome $Y$, à partir d'une cellule isolée au stade 6 à 10 cellules (Handyside et al, 1989). Dernièrement, Holding et Monk (1989) ont pu établir un diagnostic de $\beta$-thalassémie en amplifiant I'ADN obtenu à partir de blastomères d'embryon de souris avant implantation.

\section{Les dangers de la contamination}

L'analyse des séquences d'ADN par PCR est tellement sensible que la moindre contamination de l'échantillon analysé, ou du matériel de laboratoire (pipettes, appareils), ou des réactifs employés sera à l'origine de faux positifs. Ceci fait que cette technique, simple d'utilisation, automatisée et à la portée de très nombreux laboratoires, est néanmoins à utiliser avec beaucoup de prudence. II faut, en particulier, introduire à toutes les étapes du processus un moyen de contrôler la qualité, et pour certaines applications adopter la façon de travailler des centres de transfusion sanguine effectuant les tests en double aveugle. Les pièces du laboratoire où sont extraits les échantillons et où se déroulent ensuite les expériences d'amplification doivent être séparées physiquement, le matériel ne transitant pas de l'une à l'autre.

\section{CONCLUSION}

Les chercheurs et les praticiens disposent maintenant de moyens extrêmement performants d'analyse des séquences géniques. Celles-ci peuvent maintenant être étudiées à partir d'infimes quantité de matériel biologique, ouvrant la voie à toute une série d'expériences auparavant impossibles et qui feront progresser les connaissances dans de nombreux domaines, comme ceux de la biologie du développement ou de la différenciation cellulaire.

\section{RÉFÉRENCES}

Amselem $S$, Nunes $V$, Vidaud $M$, Estivill $X$, Wong C, Dauriol L, Vidaud D, Galibert F, Baiget $M$, Goossens $M$ (1988) Determination of the spectrum of $\beta$-thalassemia genes in Spain by use of dot-blot analysis of amplified $\beta$-globin DNA. Am J Hum Genet 43, 95-100 
Attree O, Vidaud D, Vidaud M, Amselem S, Lavergne JM, Goossens M (1989) Mutations in the catalytic domain of human coagulation factor IX: rapid characterization by direct sequencing of DNA fragments displaying an altered melting behavior. Genomics 4, 266-272

Bugawan TL, Saiki RK, Levenson $\mathrm{CH}$, Watson $\mathrm{RM}, \mathrm{Ha} E$ (1988) The use of non-radioactive oligonucleotide probes to analyse enzymatically amplified DNA for prenatal diagnosis and forensic HLA typing. Biotechnology 6, 943-947

Byrne BC, Li JJ, Sninsky J, Poiesz B (1988) Detection of HIV-1 RNA sequences by in vitro DNA amplification. Nucleic Acids Res 16, 4165

Chamberlain JS, Gibbs RA, Ranier JE, Nguyen PN, Caskey CT (1988) Deletion screening of the Duchenne muscular dystrophy locus via multiplex DNA amplification. Nucleic Acids Res 16, 11141-11156

Crescenzi M, Seto M, Herzig GP, Weiss PD, Griffith RC, Korsmeyer SJ (1988) Thermostable DNA polymerase chain amplification of t(14-18) chromosome breakpoints and detection of minimal residual disease. Proc Natl Acad Sci USA 85, 4869-4873

Diaz-Chico JC, Yang K, Yang K, Efremov DG, Stoming TA, Huisman THJ (1988) The detection of $\beta$-globin gene mutations in $\beta$ thalassemia using oligonucleotide probes and amplified DNA. Biochim Biophys Acta 949, 43-48

Engelke DR, Hoener PA, Collins FS (1988) Direct sequencing of enzymatically amplified human genomic DNA. Proc Natl Acad Sci USA 85, 544-548

Feldman $G L$, Williamson $R$, Beaudet $A L$, O'Brien WE (1988) Prenatal diagnosis of cystic fibrosis by DNA amplification for detection of KM-19 polymorphism. Lancet ii, 102

Frohman MA, Dush MK, Martin GR (1988) Rapid production of full-length cDNAs from rare transcripts: amplification using a single gene-specific oligonucleotide primer. Proc Natl Acad Sci USA 85, 8998-9002

Gyllensten UB, Erlich HA (1988) Generation of single-stranded DNA by the polymerase chain reaction and its application to direct sequencing of the HLA-DQA locus. Proc Natl Acad Sci USA 85, 7652-7656

Handyside AH, Pattinson JK, Penketh RJA, Delhanty JDA, Winston RML, Tiddenham EGD (1989) Biopsy of human preimplantation em- bryos and sexing by DNA amplification. Lancet i, 347-349

Higuchi R, Beroldingen $\mathrm{CH}$, von Sensabaugh GF, Erlich HA (1988) DNA typing from singles hairs. Nature 322, 543-546

Holding C, Monk M (1989) Diagnosis of betathalassemia by DNA amplification in single blastomeres from mouse preimplantation embryos. Lancet ii, 532-535

Jeffreys AJ, Wilson V, Neumann R, Keyte J (1988) Amplification of human minisatellites by the polymerase chain reaction: towards DNA fingerprinting of single cells. Nucleic Acids Res 16, 10953-10971

Kawasaki ES, Clark SS, Coyne MY, Smith SD, Champlin R, Witte ON, McCormick FP (1988) Diagnosis of chronic myeloid and acute lymphocytic leukemias by detection of leukemiaspecific mRNA sequences amplified in vitro. Proc Natl Acad Sci USA 85, 5698-5702

Kogan SC, Doherty M, Gitschier J (1987) An improved method for prenatal diagnosis of genetic diseases by analysis of amplified DNA sequences: application to hemophilia A. $N$ Engl J Med 317, 985-990

Lee MS, Chang KS, Cabanilas F, Freireich EJ, Trujillo JM, Stass SA (1987) Detection of minimal residual cells carrying the t(14-18) by DNA sequence amplification. Science 237, 175-178

Li H, Gyllensten UB, Cui X, Saïki RK, Erlich HA, Arnheim N (1988) Amplification and analysis of DNA sequences in single human sperm and diploid cells. Nature 335, 414-417

Mullis KB, Faloona F (1987) Specific synthesis of DNA in vitro via a polymerase-catalyzed chain reaction. Methods Enzymol 115, 335350

Myers RM, Maniatis T, Lerman LS (1987) Detection and localization of single base changes by denaturing gradient gel electrophoresis. Methods Enzymol 155, 501-527

Ou CY, Kwok S, Mitchell SW, Mack DH, Sninsky JJ, Krebs JW, Feorino P, Warfield D, Schochetman F (1988) DNA amplification for direct detection of HIV-1 in DNA of peripheral blood mononuclear cells. Science 239, 295297

Pignon JM, Henni T, Amselem S, Vidaud M, Duquesnoy $P$, Vernant JP, Kuentz M, Cordonnier C, Rochant H, Goossens M (1989) Frequent detection of minimal residual disease by use of the polymerase chain reaction in long-term survivors after bone marrow trans- 
plantation for chronic myeloid leukemia. Leukemia in press

Rochiltz CF, Scott GK, Dodson JM, Benz CC (1988) Use of the polymerase chain reaction technique to create base-specific ras oncogene mutations. DNA 7, 515-519

Saiki RK, Scharf F, Faloona F, Mullis KB, Horn GT, Erlich HA, Arnheim N (1985) Enzymatic amplification of $\beta$-globin genomic sequences and restriction site analysis for diagnosis of sickle cell anemia. Science 230, 1350-1354

Saïki RK, Bugawan TL, Horn GT, Mulis KB, Erlich HA (1986) Analysis of enzymatically amplified $\beta$-globin and HLA-DQ $\alpha$ DNA with allele specific oligonucleotide probes. Nature 324, 163-166
Saïki RK, Chang CA, Levenson CH, Warren TC, Boehm CD, Kazazian HH, Erlich HA (1988) Diagnosis of sickle-cell anemia and $\beta$ thalassemia with enzymatically amplified DNA and nonradioactive allele-specific oligonucleotide probes. $N$ Engl J Med 319, 537541

Wong D, Dowling CE, Saïki RK, Higuchi RG, Erlich HA, Kazazian HH Jr (1987) Characterization of $\beta$-thalassaemic mutations using direct genomic sequencing of amplified single copy DNA. Nature 330, 384-386

Wrischnick LA, Higuchi RG, Stoneking M, Erlich $H A$, Arnheim N, Wilson AC (1987) Length mutations in human mitochondrial DNA: direct sequencing of enzymatically amplified DNA. Nucleic Acids Res 15, 529-542 